\title{
Environmental hazards and restraining methods of pavement asphalt
}

\author{
Jing Zhang, ${ }^{1, *}$, Zhiqing $\mathrm{Guo}^{2}$, and Yupeng $\mathrm{Cao}^{3}$ \\ ${ }^{1}$ Shandong University of Science and Technology, College of Transportation, 266590 Qingdao,China \\ ${ }^{2}$ Weifang Lutong Engineering Construction Co., Ltd, 261061 Weifang, China \\ ${ }^{3}$ Weifang University, College of Civil Engineering and Architecture, 261061 Weifang, China
}

\begin{abstract}
There are environmental problems such as pollutant emission in the construction and service of road asphalt pavement. In order to explore the hazards of road asphalt to the environment, the types of pollutants from the construction and use of road asphalt pavement are analyzed. The various harmful components of pavement asphalt materials and their influencing factors are discussed. The corresponding pollution inhibition measures are introduced. The existing research work of asphalt pavement pollution is analyzed and suggestions for future research are proposed.
\end{abstract}

\section{Introduction}

Asphalt pavement has become the main form of highway pavement in our country. However, pavement asphalt has brought a series of ecological problems during its production, construction and operation stage. With more and more attention paid to environmental protection, the ecological environmental protection of highway asphalt has aroused the common concern of road and environmental workers. Therefore, it is necessary to conduct a scientific and reasonable analysis and research on the pollution emission of pavement asphalt, and clarify the impact of pavement asphalt components on human health and the environment. For this reason, this article used the method of literature survey to discuss the potential hazards of road asphalt to air, water and soil, introduced the main suppression methods of harmful smoke and leachate during the operation of road asphalt paving, and gave the directions for improving and alleviating asphalt pollution

\section{Research on the hazards of road asphalt and treatment measures}

\subsection{Research on the hazards of road asphalt to the air and treatment measures}

In the construction phase of asphalt pavement, its volatile components migrate into the air in the form of smoke to form asphalt smoke. Asphalt smoke is a special pollutant with

\footnotetext{
*Corresponding author: 3288424787@qq.com
} 
complex composition generated during production and construction. It usually exists in the air in the form of aerosol and contains a large amount of polycyclic aromatic hydrocarbon compounds (PAHs) and a small amount of oxygen, sulfur, heterocyclic mixture of nitrogen.

[1] The components of asphalt smoke are extremely complex and vary with the source of asphalt. ${ }^{[2]}$

It has been confirmed that the asphalt smoke emitted by road mixing contains more than ten carcinogens, but the carcinogenicity of asphalt smoke has not been confirmed. The research on harmful components of asphalt smoke mainly focuses on PAHs. The report of the International Agency for Research on Cancer (IARC) pointed out that the 2 to 3 ring polycyclic aromatic hydrocarbons have a stimulating effect, and the 4 to 6 ring polycyclic aromatic hydrocarbons have carcinogenic effects. For asphalt smoke, 3-7 rings polycyclic aromatic hydrocarbons are the main mutagenic substances. With the increase of heating temperature, the concentration of PAHs in the 4-6 rings will also increase. Asphalt smoke has a stimulating effect on human skin and mucous membranes. People who are exposed to high concentrations of asphalt smoke for the first time or who are sensitive to asphalt smoke can easily cause dermatitis, conjunctivitis, rhinitis, pharyngitis and other diseases.

Road asphalt is solid or semi-solid at room temperature. The PAHs contained in it have extremely limited volatilization at the storage and construction temperature. However, when the asphalt generated during paving and construction on poorly ventilated sections such as tunnels and urban roads, the flue gas will have a high concentration, and its harmful components will have varying degrees of impact on the health of workers. ${ }^{[3]}$

At present, it is generally believed that the release of road asphalt smoke occurs in hightemperature operations such as heating mixing and paving. In order to mix asphalt and aggregates uniformly during road construction, heating must be used to reduce the viscosity of asphalt. When the asphalt is heated unevenly, asphalt smoke will be formed.

The amount of asphalt smoke produced is affected by temperature and the amount of carrier gas. At present, the majority of scholars agree that as the temperature increases, the emissions of asphalt smoke and the production of polycyclic aromatic hydrocarbons increase; under hypoxic conditions, the amount of polycyclic aromatic hydrocarbons in the asphalt smoke increases. ${ }^{[4]}$ Asphalt mixing speed, asphalt oil source and type, and asphalt moisture content are also key factors affecting the release of asphalt smoke. ${ }^{[5]}$ However, the current analysis of the factors affecting the release of asphalt smoke mainly focuses on the change of a single factor, and there are few studies on the superimposed effects of many factors leading to the release of asphalt smoke.

For the treatment of asphalt smoke during pavement construction, the main methods of modifying the base asphalt and reducing the mixing temperature of the asphalt mixture are used to inhibit the generation of asphalt smoke. ${ }^{[6]}$

Asphalt modification technology is to add polymer modifiers, adsorbents, flame retardants and other asphalt smoke inhibitors to the asphalt. Warm mixing technology and emulsification technology suppress the volatilization of asphalt smoke by reducing the mixing temperature of the asphalt mixture.

Although the modification of asphalt can reduce the amount of smoke emission to a certain extent, most of the additives will affect the performance of the asphalt, and the treatment cost is difficult to control. As the basic pavement material for road engineering construction, asphalt has important social and economic benefits in terms of performance and cost. In the treatment of asphalt flue gas, only ensuring the performance of the asphalt and reducing the cost of treatment can facilitate the application and promotion of related technologies. 


\subsection{Research on the hazards of road asphalt to water bodies and treatment measures}

Asphalt pavement has the opportunity to directly contact with water. During the use of the pavement, due to the load of tires and the erosion of dynamic water, some components in the asphalt will enter the water body with the seepage of water, forming asphalt leachate, causing asphalt water pollution. The asphalt leachate is mainly formed by rain washing and soaking. When the asphalt pavement suffers from early damage such as water damage, rutting, looseness, aging, oiling, etc., the asphalt and aggregate will be peeled off to form fine asphalt particles. The asphalt particles will enter the water body through external effects such as wind blowing or rain washing.

A large number of tests have shown that organic solids, PAHs and phenols in road surface asphalt can leach out of the asphalt and cause pollution to water bodies. In terms of the sources of pollutants from road surface runoff, it is generally believed that the pollutants from asphalt pavements or asphalt particles worn out by tires are toxic organic PAHs.

In order to simulate the leaching process of polycyclic aromatic hydrocarbons in asphalt, some scholars conducted indoor leaching tests. It was found that the concentration of PAHs in the bitumen leachate from different oil sources varied greatly. For asphalt pavements, the concentration of PAHs in the asphalt leaching solution is usually lower than the surface water limit, but for asphalt pavements with a certain age, the APHs in the asphalt leaching solution is higher than that of fresh asphalt.

External conditions will also affect the leaching performance of asphalt components in water. It is generally believed that the asphalt particles is smaller, the surface area and the water contact surface is larger. The chance of organic matter contacting water increases, and its concentration increases. ${ }^{[7]}$ Temperature affects the nature and state of asphalt materials in the pavement structure, and the distribution coefficient of organic matter in the liquid phase (water phase) and solid phase (asphalt phase), thereby changing the amount of asphalt components leached in water. Acid rain will change the nature of rainwater to a certain extent, increase the chemical activity of water, and then change the dispersion of asphalt in water. Studies have found that weak acid can promote the leaching of components in asphalt. ${ }^{[8]}$ The presence of certain chemical substances such as Organic solvents may also increase the dissolution and release of asphalt in water bodies.

In view of the aggregation and diffusion behavior of asphalt molecules in the leachate at the micro level, relevant scholars have used molecular dynamics simulation (MD) to study asphalt aggregation behavior and asphalt-aggregate interface interaction. In terms of asphalt aggregation behavior, a Yen model of the process of asphalt molecules from monomer molecules to flocculation phase separation was constructed through molecular simulation software; in terms of asphalt-aggregate interface interaction, a molecular structure model of asphalt-aggregate interface has been established, which are used to estimate interface damage. ${ }^{[9]}$

Although molecular dynamics has made some progress in studying asphalt molecules, there are very few studies on the migration or diffusion of asphalt molecules in the asphaltwater interface. At the same time, the leaching test of road asphalt lacks a uniform and suitable test method, which makes it difficult to propose specific measures to prevent or control the leaching pollution of harmful components of asphalt. In view of the potential hazards of road asphalt to water bodies, the organization and management and process control during pavement construction should be strengthened, and the processing and use of asphalt materials should be reasonably guided to avoid or reduce the pollution of asphalt to the water environment. 


\subsection{Research on the hazards of road asphalt to soil and treatment measures}

In the course of road use, the abraded bituminous components are deposited and adsorbed to migrate to the soil, causing harm to the soil environment. Studies have shown that the harm of asphalt pavement to the soil mainly results in the pollution of heavy metals and PAHs in the soil. Among them, road dust and heavy metals mainly come from automobile exhaust emissions and dust deposition ${ }^{[10]}$, and PAHs mainly comes from asphalt pavement corrosion and asphalt particles under wear.

There are few concentrated studies on the analysis of PAHs in roadside soil, but related reports also mention that the soil on both sides of the asphalt road traffic trunk is polluted by PAHs to varying degrees. Studies in developed countries also show that in the past 100150 years, the concentration of PAHs in soil (especially in urban areas) has been increasing, and PAHs are mostly in the 4-6 rings. ${ }^{[11]}$ During the use of asphalt pavement, PAHs will also compound with the heavy metals existing in the soil on both sides of the road, increasing the degree of soil pollution.

The sources of PAHs in roadside soils mainly include atmospheric deposition, road surface runoff, and migration of worn-out asphalt particles. PAHs discharged into the atmosphere are adsorbed on the smoke and dust in a molecular state, and sink with the smoke and dust in the air. A part of the small particles agglomerated with each other and became larger particles and naturally settled down; the other part of the small particles of smoke and dust staying in the air fell to the ground with raindrops. In addition, the asphalt particles under the corrosion and wear of the asphalt pavement will move to the soil along the road surface with the surface runoff under the action of tire load and dynamic water erosion. After surface leaching, the soil will also be polluted by PAHs polycyclic aromatic hydrocarbons.

Targeting 16 optimal control PAHs proposed by the U.S. Environmental Protection Agency, the monitoring research on the content and distribution of PAHs in the soil along traffic roads shows that the greater the traffic volume on the road, the longer the traffic time, the more heavy vehicles, the more traffic along the road, the content of high-ring PAHs in the soil will be higher. The content of PAHs in the soil on both sides of the traffic road decreases as the distance from the traffic road increases. ${ }^{[12]}$

In order to ensure the road performance of asphalt mixtures, reducing the pollution of asphalt to the soil along traffic roads is rarely improved by asphalt itself, but to avoid corrosion and wear of asphalt pavements by reducing overweight, overloading and limiting traffic and improving pavement quality. In addition, protection forests along the traffic line can absorb PM2.5 and reduce heavy metal pollution, thereby causing a absorption and blocking effect on organic compounds such as PAHs in the soil. Some plant crops with fibrous root roots also have a certain absorption effect on PAHs. Alfalfa, magnolia, rye seedlings, etc. can be used to absorb and degrade some PAHs to prevent the restoration of soil contaminated by PAHs.

\section{Summary and outlook}

Pavement asphalt's harm to air, water and roadside soil is mainly through PAHs pollution. Although indoor tests show that asphalt PAHs pollution is generally lower than the air and groundwater limit indicators, when the asphalt mixture is mixed and constructed under relatively closed conditions, PAHs precipitates more serious, and in the later stage of asphalt pavement use, more PAHs will be leached from the waste asphalt pavement, which will cause harm to the water body and roadside soil.

The construction and service of asphalt pavement are in an open environment, and the treatment of harmful components of the asphalt is difficult, and there is still no better 
source treatment method. At present, most researches try to suppress asphalt pollution through asphalt modification and improvement of paving technology. As for the treatment of asphalt fume during pavement mixing construction, the main focus is on the development of modified asphalt, and the cost of treatment is difficult to control; for the suppression of asphalt leachate during the service phase of the pavement, more efforts should be made to optimize the organization and control, and start from rational and scientific construction to reduce cross-contamination of asphalt components; for the treatment of asphalt soil pollution, crops that can absorb and degrade PAHs can be planted along the road to prevent and repair the soil contaminated by asphalt components.

\section{References}

1. H. Cai, P. Wang, T. Wang, Y. Zhang, Summary of research on environmental pollution of potentially harmful components in asphalt [J].Petroleum Asphalt, 23(02),1-8(2009)

2. J. Kurek, A. Kriech, H. Wissel, L. Osborn, Laboratory generation and evaluation of paving asphalt fumes [J]. Journal of the Transportation Research Board, 35 40(2007)

3. J. Zheng, Z. Zhou, D. Zhang, Y. Zhang, X. Yang, Study on TSP and Polycyclic Aromatic Hydrocarbons Pollution in the Air During Asphalt Paving of Tunnel Pavement $[\mathrm{J}]$.Environmental Monitoring Management and Technology, 22(06),3941(2010)

4. E. Gasthauer, M. Maze, J. Marchand, J. Amouroux, Characterization of asphalt fume composition by GC/MS and effect of temperature [J]. Fuel, $87,1428 \sim 1434(2008)$

5. G. Huang, Z. He, T. Huang, Indoor measurement and influencing factors analysis of asphalt smoke release at high temperature[J].Journal of Building Materials, 18(02),322-327(2015)

6. C. Liu, Z. Li, A. Ning, G. Cheng, Analysis and Prospect of Asphalt Flue Gas Treatment Technology[J].Petroleum Asphalt, 34(01),41-45(2020)

7. H. Cai, P. Wang, Y. Zhang, Study on the leaching characteristics of potentially harmful components in asphalt[J].Materials Guide, 24(S1),450-453(2010)

8. Y. Lu, L. Wang, Nanoscale modeling of the mechanical properties of asphalt and aggregate [J].Pavements and Materials, 43- 53(2010)

9. A. Ailaiti, M. Aizezi, W. J, G. Aini, Ai. Maimat, Alimujiang Kasmu. Road plot in Korla, Xinjiang Dust and Heavy Metal Pollution and Health Risk Assessment $[$ J].Geology in China, 47(06), 1915-1925(2020)

10. L. Xiong, J. Wu, M. Wang, Q. Tang, C. Sha, D. Li, H. Tang, J. Wu, Summary of soil polycyclic aromatic hydrocarbon pollution and risk prevention and control along traffic roads[J].Acta Eco-Environmental Sciences, 27(05),974-982(2018)

11. H. Duan, Multimedia PAHs pollution and comprehensive health risk analysis of road environment [D]. Henan University( 2016)

12. C. Yang, J. Hu, J. Guo, Z. Chen, Pollution characteristics and source analysis of polycyclic aromatic hydrocarbons in PM_(2.5) in Guiyang in winter and summer[J].Environmental Chemistry, 33(01),161-162(2014) 